\title{
Stromal CD4+ and CD8+ T Cells in Human Breast Carcinomas. Its Correlation with Chemokine MIG/CXCL9
}

\author{
Graciela Laguens, Silvia Coronato, Jorge Chambó, Vanda Di Girolamo \\ Department of Pathology, School of Medicine, National University of La Plata, \\ La Plata, Argentina \\ Email: vandadigirolamo@gmail.com
}

Received May 2, 2012; revised June 7, 2012; accepted June 30, 2012

\begin{abstract}
The interaction between some chemokines with tumoral and stromal cells can influence tumor progression. CXCL9, a monokine induced by interferon gamma (MIG), targets lymphocytes. The aim of our study was to identify and quantify CD4+ and CD8+ T cells in the stroma of human breast cancer and correlate them with the presence of MIG/CXCL9. In 58 specimens of human breast carcinoma and 10 normal breast tissue from mammoplasty surgery, immunohistochemistry and ELISA methods were performed. The number of CD4+ and CD8+ T cells in breast cancer tissue was significantly increased compared with normal breast tissue with a clear predominance of CD8+ T cells. MIG/CXCL9 levels were significantly elevated respect normal breast tissue. This chemokine correlated with the number of CD8+ T cells only in non-metastatic tumors. These data suggest that MIG targets cytotoxic T cells. Their recruitment into breast carcinoma can play a critical role in malignant progression, inhibiting the production of metastasis.
\end{abstract}

Keywords: Breast Cancer; CD4+ T cells; CD8+ T Cells’ MIG/CXCL9; Chemokines

\section{Introduction}

Tumor infiltrating lymphocytes (TIL) are a common finding in almost tumors. In breast cancer, one of the tumors with major impact in women's health, these immune cells infiltrate the stroma indicating a cellular immune response of uncertain result. It remains unclear weather immune response influences the tumor growth and so as the mechanisms involved in immune cells recruitment. Nevertheless, kinetics experimental studies of tumor infiltrating lymphocytes in mice challenged in the mammary region with an aggresive tumor cell line demonstrated that $\mathrm{T}$ cells are recruited in stroma of murine breast tumor after the first week of the challenge. Gradually CD4+ T cells subpopulation diminishes and an increase of CD8+ $\mathrm{T}$ cells is observed. The presence of these lymphocytes was considered the expression of an antitumor immune response, capable of preventing progression and metastasis [1].

In cancer progression, tissue homeostasis is perturbed. Stroma resident cells and tumor cells locally secrete soluble factors such as proteases, growth factors, cytokines and chemokines favouring the recruitment of cells such as $\mathrm{T}$ cells, mast cells, macrophages and dendritic cells [2]. Chemokines represent a family of small mo- lecular weight chemotactic cytokines regulating leukocyte trafficking and other biological functions such as angiogenesis/angiostasis [3,4].

It is known that in breast cancer, tumor cells produce CCL5/RANTES and the level of expression correlates with the extent of macrophage infiltration and lymph node metastasis. In esophageal carcinoma, CCL2/MCP1 expression is correlated with the level of macrophage infiltration, tumor angiogenesis and invasion [5]. These and others acumulating data show that chemokines can serve as tissue specific attractants molecules for recruitment of immune cells in cancer tissue.

Given that chemokines play an important roles in multiple steps of tumor progresssion and metastasis, a chemokine candidate suggested as a potential recruitment factor includes interferon-induced MIG/CXCL9 member of the non-ERL-CXC chemokine family [6]. This chemokine is secreted by stromal cells, macrophages, dendritic cells and B cells. MIG/CXCL9 interact with CXCR3 receptor. This receptor is highly expressed on $\mathrm{T}$ helper-1 (Th-1) cells, effector CD8+ T cells and innatetype cells such as natural killer (NK), macrophages and dendritic cells. All these cells are recruited to tumoral stroma and MIG/CXCL9 specifically can interact with T cells [7-9]. 
It is demonstrated that in human gastric and colorectal carcinoma signifinat levels of CXCL9 and CXCL10 are produced by stromal cells and are correlated with CXCR3-positive TIL [10]. These carcinomas are associated with a good prognosis [11].

Over the last years there are few works studying the presence of MIG/CXCL9 in human breast carcinomas in the bibliography $[2,3,12]$. On the other hand, in murine models the role of MIG/CXCL9 in growth suppression of breast tumors as well as metastasis establishment is widely proven $[13,14]$.

The aim of our study was to identify and quantify the presence of CD4+ and CD8+ T cells in the stroma of human breast cancer and examine the correlation between these lymphocytes and MIG/CXCL9 with the objective of providing new insights on microenvironment contribution to tumor progression.

\section{Materials and Methods}

\subsection{Patients}

Tumor tissues were surgically obtained from 58 female patients, ranging in age from 43 to 75 years, with primary infiltrating breast carcinoma. They were all treated by wide excision of the tumor or total mastectomy. Histopathological types and tumor stages were classified according the International System of Nothinghan. Histological diagnosis was performed using routine methods. It should be emphasized that none of patients received chemotherapy before the study and none of them presented evidence of local o systemic infection. As control 10 samples of normal breast tissue from mammoplasties were used. After excision, each specimen was fixed in $10 \%$ buffered formalin for paraffin embedding. Simultaneously each specimen was divided into two parts: half of the tissue was snaped and cryostat sections for immunohistochemical staining with monoclonal antibodies (mAb) applicable only on frozen sections. The remaining half was stored in liquid nitrogen to be used for MIG chemokine determination by ELISA (Enzime Linked Immunosorbent Assay). The methods performed did not have influence on the diagnostic and therapeutic procedures for patients and there was no conflict with the ethical requirements of the Bioethic Commitee of our hospital.

\subsection{Immunochemistry}

Frozen sections were employed to detect CD4+ and CD8+ $\mathrm{T}$ cells with mAb CD4+ ( $\mathrm{T}$ cell helper/inducer BioGenex) and mAb CD8 ( $\mathrm{T}$ cell supressor/cytotoxic BioGenex). After incubation with the primary antibody, the sections were washed in PBS and incubated with biotynilated link antibody. The estreptavidin-peroxidase complex (Vectastain ABC KIT, Peterborough, England) was used to immunohistochemical staining and carbazol chromogen was used for visualization. Finally, sections were counterstained with Mayer haematoxylin (BioPack, MR, Buenos Aires, Argentina) and mounted with glycerol-vinyl aqueous solutions (Zymed Lab. Inc. San Francisco, USA).

\subsection{ELISA Analysis}

Tumor tissues and control tissues were mechanically homogenized with a micro blender immersed in an ice bath with a saline solution (PBS) balanced at 7.4 pH in a 1:2 relation; tissue volume/PBS volume. Homogenates were centrifuged for 1 hour at $8.000 \mathrm{rpm}$ at $4^{\circ} \mathrm{C}$. Supernants were used for determining the presence of MIG by ELISA. Anti-human MIG (mAb) and goat anti-human polyclonal biotinylated murine monoclonal antibodies were used following the manufacturers' instructions. Briefly each ELISA plate was covered with anti-MIG (mAb) in a concentration of $2 \mu \mathrm{g} / \mathrm{ml}$ with PBS overnight at $37^{\circ} \mathrm{C}$. After washing with PBS, each well was filled with blocking solution (PBS with $1 \%$ albumin bovine and 5\% sucrose) and was incubated for 3 hours at room temperature. After washing with PBS the wells were covered with $100 \mu \mathrm{l}$ of supernatant of normal and tumoral tissues and the plate was incubated at $37^{\circ} \mathrm{C}$ for 2 hours. After washing three times with PBS $100 \mu \mathrm{l}$ of polyclonal biotinylated anti-MIG were added to each well and incubated for 1 hour at $37^{\circ} \mathrm{C}$. The plates were washed 3 times with PBS and $100 \mu$ l of a 1:1000 dilution of conjugate of streptavidine and peroxidase were added to each well. After a 30 -min incubation at $37^{\circ} \mathrm{C}$, the plate was washed three times with PBS, $100 \mu$ l of a $1: 1 \mathrm{mix}-$ ture of $\mathrm{H}_{2} \mathrm{O}_{2}$ with tetramethylbenzidine were added and incubated at room temperature after 30 minutes. The reaction was stopped with $50 \mu \mathrm{l}$ of $\mathrm{H}_{2} \mathrm{SO}_{4}, 2 \mathrm{M}$. Absorbance was measured using a microplate reader at $450 \mathrm{~nm}$.

Results are expressed as quotient stated as follows:

$$
\text { Quotient }=\frac{\text { Absorbance of tumor tissue }}{\text { Mean absorbance of normal tissues }+2 \text { SD }}
$$

Results greater or equal to 1.0 are considered to be positive for the presence of MIG.

\subsection{Computerized Image Analysis}

T cells were counted using a digital image analyzer (ImagePro v6.3 Media Cybernetics, USA). For this purpose immunohistochemically stained cells were recognized using the color cube option of the count/size function of the software. For that purpose cell-containing tissue images were captured using a digital camera (Olympus DP71, Olympus, Japan). Ten images $(1360 \times 1024$ resolution, spatial resolution up to $0.16 \mu \mathrm{m} /$ pixel, RGB and 
TIFF format) were captured from each sample. The total number of cells was registered in 10 images. Data obtained were exported to a spreadsheet to perform a statistical analysis.

\subsection{Statistical Analysis}

Data obtained were statistically analyzed by Student's t test and Spearman's correlation test. $\mathrm{P}<0.05$ values were considered significant.

\section{Results}

The stroma of all breast cancers showed a great number of CD4+ and CD8+ T cells $\left(2544 \pm 540 \mathrm{cel} / \mathrm{mm}^{2}\right.$ and $6872 \pm 970 \mathrm{cel} / \mathrm{mm}^{2}$, respectively). Their number was significantly increased with respect to control normal breast tissue (CD4+ cells: $1130 \pm 122 \mathrm{cel} / \mathrm{mm}^{2}$ and CD8+ cells: $\left.457 \pm 98 \mathrm{cel} / \mathrm{mm}^{2}\right)$. Additionally, in breast tumors an inverted CD4+: CD8+ ratio (1:2) between these lymphocytes subsets was observed (normal breast 2:1 ratio). Figure 1

In $84 \%$ of tumors the presence of MIG/CXCL9 was detected, expressed as it was indicated in materials and methods (quotient average $=2.12 \pm 1.17$ ). In the remaining $16 \%$ the quotient was lower than 1 and considered negative. There was a low expresión of MIG/CXCL9 in normal breast tissues (Figure 2).

In tumors with metastasis there was no correlation between the presence of CD4+ and CD8+ T cells and MIG/CXCL9 expression. In tumors without metastasis there was a significantly positive correlation between the number of CD8+ $\mathrm{T}$ cells and Mig/CXCL9 ( $<<0.02)$ (Figure 3).

\section{Discussion}

In breast cancer, tumor growth is modulated by a complex interaction between tumor cells and immune cells, cytokines and chemokines [15]. Despite the presence of $\mathrm{T}$ cells in tumor microenvironment, antitumor immune response is ineffective and the tumor growths and metastasizes. The function of those lymphocytes seems to be suppressed. Multiple mechanisms of immune supression that act in the tumor microenvironment have been proposed and several of these have been validated to be functionally important in preclinical studies [16]. For instance, preliminary data have suggested that melanoma metastasis that contain CD8+ $\mathrm{T}$ cells show greater presence of FoxP3+ regulatory $\mathrm{T}$ cells, as well a higher expression of PD-1 and IDO (Indoleamine-2,3-dioxigenase), supporting the notion that the tumor microenvironment can be rich in suppressive mechanisms that could contribute to cell disfunction [17]. However, there are data showing that in some gastric tumors, the presence of great number of tumor-infiltrating lymphocytes is

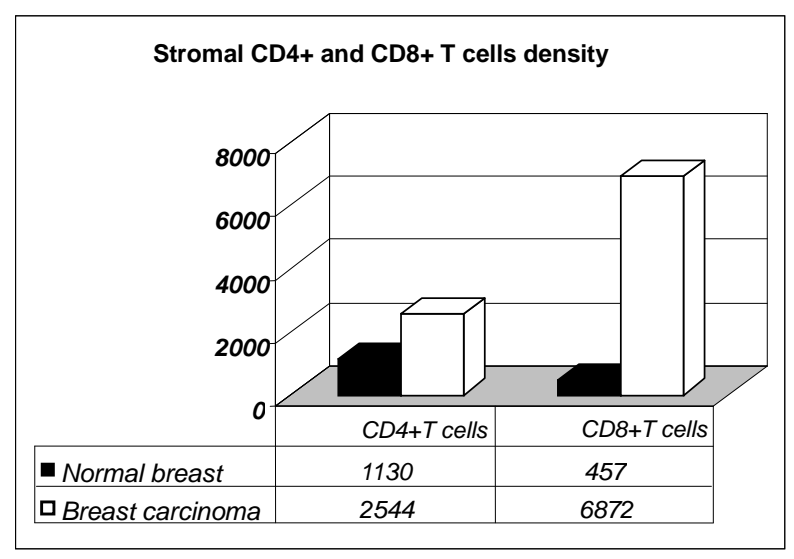

Figure 1. Increased number of CD4+ and CD8+ $T$ cells in the stroma of breast cancer as compared with normal breast tissue with an inverted ratio.

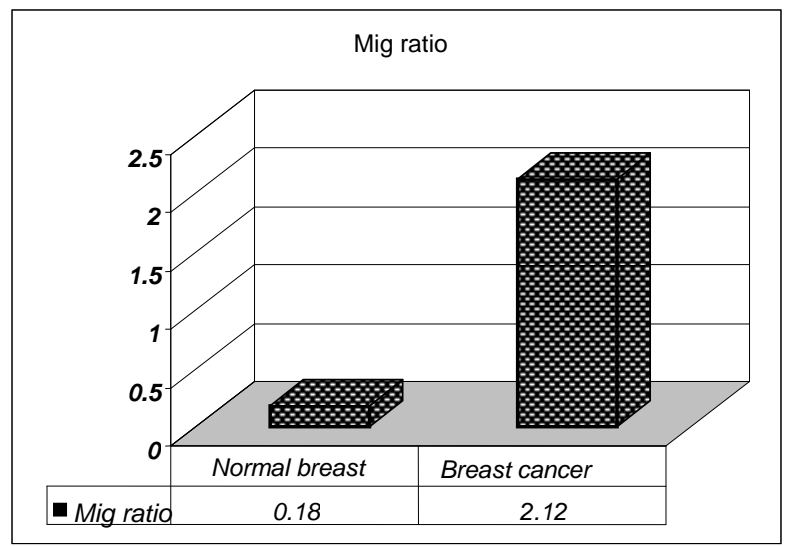

Figure 2. Presence of MIG/CXCL9 in breast cancer as compared with normal breast tissue.

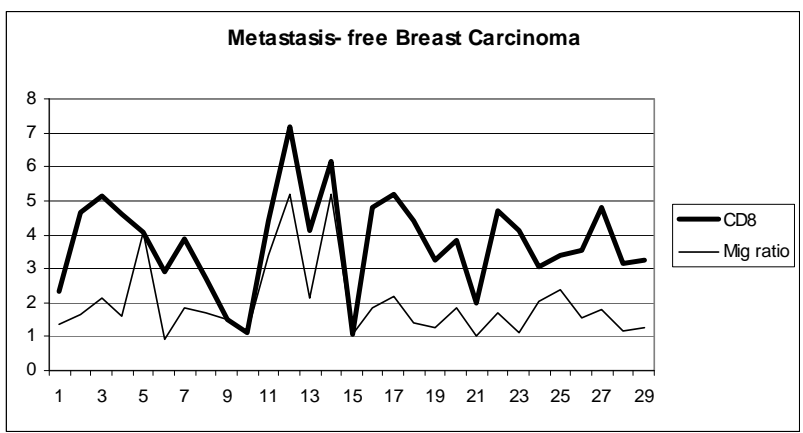

Figure 3. Positive correlation between MIG/CXCL9 and stromal CD8+ $T$ cells in breast carcinoma without lymph node metastasis.

associated with better prognosis. The majority of these lymphocytes were positive for CXCR3 receptor and its ligand MIG/CXCL9 was abundantly expressed by stromal cells and a small number of cancer cells [18,19]. MIG/CXCL9 expression promotes an immune cell infíltrate which could have the capacity to control tumor 
growth and metastasis [20]. For instance, in renal cell carcinoma the increase of MIG/CXCL9 expression is associated with a more favourable prognosis. Its role in $\mathrm{T}$ cell recruitment and its antitumorigenic activity was demonstrated [21]. Other studies have shown that when the MIG/CXCL9 level in the tumor microenvironment is high, the metastatic capacity of tumor cells is considerably reduced [20].

Our results indicate that there are an increase of both CD4+ and CD8+ $\mathrm{T}$ cells in the stroma of malignant breast tumors. The number of CD4+ $\mathrm{T}$ cells doubles those observed in normal breast tissues, whereas the number of CD8+ $\mathrm{T}$ cells increases more than ten times. The relationship between both subpopulations is inverted in favour of CD8+ T cells.

Similar findings were observed by us in lymph nodes axillary without metastasis draining human breast tumors [22] and they are described by other authors [23,24].

The presence of MIG/CXCL9 has been detected in 84 $\%$ of human breast carcinomas, whereas in normal breast tissues there was no expression. The increase of MIG/CXCL9 level was similar in tumors with and without metastasis. In both cases there was also a significant increase of the number of CD4+ and CD8+ T cells compared with control. In metastasis-free breast cancer a significative correlation was found between the number of CD8+ T cells and MIG/CXCL9. However, in breast cancer with metastasis the total number of lymphocytes did not correlate with the presence of MIG/CXCL9. Nevertheless, our results show that the presence of MIG/CXCL9 is correlated with CD8+ T cells rather than with CD4+ $\mathrm{T}$ cells in those tumors without metastasis. These tumor-specific CD8+ T cells can interfere with tumor progression. This CD8+-MIG/CXCL9 correlation is not observed in tumors with metastasis. Other authors also found that CD8+ T cells negatively affects metastasis establishment in breast tumors [25]. It was demonstrated that the absence of MIG/CXCL9 can limit the migration of activated T CD8+ cells, which in turn could limit the effectiveness of antitumor response in melanoma metastasis [17].

In conclusion, consistent with the influence of the microenvironment on tumor progression, the demonstration of a positive correlation of the chemokine MIG and supressor/cytotoxic T cells opens a new pathway to a better knowledge on $\mathrm{T}$ cells recruitment in tumoral stroma. This study expands our current understanding of MIG/CXCL9 in tumor biology and renders further support to chemokines strategies to control breast cancer behavior.

\section{REFERENCES}

[1] J. B. Reome, J. C. Hylind, R. W. Dutton and M. J. Dobrzanski, “Type 1 and Type 2 Tumor Infiltrating Ef- fector Cell Subpopulations in Progressive Breast Cancer,” Clinical Immunology, Vol. 111, No. 1, 2004, pp. 69-81.

[2] D. G. De Nardo and L. M. Coussens, "Balancing Immune Response: Crosstalk between Adaptative and Innate Immune Cells during Breast Cancer Progression,” Breast Cancer Research, Vol. 9, No. 4, 2007, pp. 212-222.

[3] A. Ben-Baruch, "The Multifaceted Roles of Chemokines in Malignancy,” Cancer and Metastasis Reviews, Vol. 25, No. 3, 2006, pp. 357-371.

[4] D. Raman, P. J. Baugher, Y. M. Thu and A. Richmond, "Role of Chemokines in Tumor Growth," Cancer Letters, Vol. 256, No. 2, 2007, pp. 137-165.

[5] T. Tanaka, Z. Bai, Y. Srinoulprasert, B. G. Yang, H. Hayasaka and M. Miyasaka, "Chemokines in Tumor Progression and Metastasis,” Cancer Science, Vol. 96, No. 6, 2008, pp. 317-322. doi:10.1111/j.1349-7006.2005.00059.x

[6] M. L. Balestrieri, A. Balestrieri, F. P. Mancini and C. Napoli, "Understanding the Immunoangiostatic CXC Chemokine Network," Cardiovascular Research, Vol. 78, No. 2, 2008, pp. 250-256.

[7] J. R. Groon and A. D. Luster, "CXC3 Ligand: Redundant, Collaborative and Antagonistic Functios," Immunology and Cell Biology, Vol. 89, 2011, pp. 207-215. doi:10.1038/icb.2010.158

[8] A. E. Karnoub and R. Weinberg, "Chemokine Networks and Breast Cancer Metastasis,” Breast Disease, Vol. 26, No. 3-4, 2007, pp. 75-85.

[9] J. Vandercappellen, J. Van Damme and S. Struyf, “The Role of CXC Chemokines and Their Receptors in Cancer,” Cancer Letters, Vol. 267, No. 2, 2008, pp. 226-244.

[10] P. Allavena, G. Germano, F. Marchesi and A. Mantovani, "Chemokines in Cancer Related Inflammation," Experimental Cell Research, Vol. 317, No. 5, 2011, pp. 664667.

[11] A. Mantovani, B. Savino, M. Locati, L. Zammataro, P. Allavena and R. Bonecchi, "The Chemokine System in Cancer Biology and Therapy,” Cytokine \& Growth Factor Review, Vol. 21, No. 1, 2010, pp. 27-39.

[12] A. Ben-Baruch, "Pro-Malignancy and Putative Antimalignancy Chemokines in the Regulation of Breast Cancer Progression,” In: F. Columbus, Ed., Focus on Immunology Research, Nova Science Publishers, New York, 2006, pp. 1-46.

[13] T. C. Walser, S. Rifat, X. Ma, N. Kundu, C. Ward, O. Goloubeva et al., "Antagonism of CXCR3 Inhibits Lung Metastasis in a Murine Model of Metastatic Breast Cancer,” Cancer Research, Vol. 66, No. 15, 2006, pp. 77017707. doi:10.1158/0008-5472.CAN-06-0709

[14] T. Walser, X. Ma, N. Kundu, R. Dorsey, O. Goloubeba, A. M. Fulton, "Immune Mediated Modulation of Breast Cancer Growth and Metastasis by the Chemokine Mig (CXCL9) in a Murine model,” Immunother, Vol. 30, No. 5, 2007, pp. 490-497.

[15] J. Valdivia-Silva, J. Franco-Barraza, A. Esparza Silva, G. Du Pont, G. Soldevila, I. Meza, et al., "Effect of Pro-Inflammatory Cytokine Stimulation on Human Breast Cancer: Implications of Chemokine Receptor Expression in 
Cancer Metastasis,” Cancer Letters, Vol. 283, No. 2, 2009, pp. 176-185.

[16] T. F. Gajewski, Y. Meng, C. Blank, et al., "Immune Resistance Orchestrated by the Tumor Microenvironment," Immunological Reviews, Vol. 213, No. 1, 2006, pp. 131145.

[17] H. Harlin, Y. Meng, A. C. Peterson, et al., "Chemokine Expression in Melanoma Metastases Associated with CD8 + T-Cell Recruitment," Cancer Research, Vol. 69, No. 3, 2009, pp. 3077-3085.

[18] H. Ohtani, Z. Jin, S. Takegawa, T. Nakayama and O. Yoshie, "Abundant Expression of CXCL9 (MIG) by Stromal Cells That Include Dendritic Cells and Accumulation of CXCR3+ T Cells in Lymphocyte-Rich Gastric Carcinoma," The Journal of Pathology, Vol. 217, No. 1, 2009, pp. 21-31.

[19] H. Ohtani and O. Yoshie, "Morphometric Analysis of the Balance between CXCR3+ T Cells and FOXP3+ Regulatory T Cells in Lymphocyte-Rich and Conventional Gastric Cancers,” Virchows Arch, Vol. 456, No. 6, 2010, pp. 615-623.

[20] A. Fulton, F. Miller, A. Weise and W. Z. Wei, "Prospects of Controlling Breast Cancer Metastasis by Immune Intervention,” Breast Disease, Vol. 26, 2007, pp. 115-127.

[21] T. Kondo, H. Nakazawa, F. Ito, Y. Hashimoto, Y. Osaka,
K. Futatsushama, et al., "Favorable Prognosis of Renal Cell Carcinoma with Increased Expression of Chemokines Associated with a Th1-Type Immune Response," Cancer Science, Vol. 97, 2006, pp. 780-786. doi:10.1111/j.1349-7006.2006.00231.x

[22] W. Di Girolamo, S. Coronato, E. Portiansky and G. Laguens, "Profile of Immune Cells in Lymph Nodes Draining Human Malignant Tumors,” Medicina, Vol. 68, 2008, pp. 423-427.

[23] S. Hirano, Y. Iwashita, A. Sasaki, S. Kai, M. Ohta and S. Kitano, "Increased mRNA Expression of Chemokines in Hepatocellular Carcinoma with Tumor-Infiltrating Lymphocytes," Journal of Gastroenterology and Hepatology, Vol. 22, No. 5, 2007, pp. 611-612.

[24] J. Yu, “Targeting Chemokines as a Therapeutic Option for Hepatocellular Carcinoma: A Reality or Just a Fantasy?” Journal of Gastroenterology and Hepatology, Vol. 22, 2007, pp. 611-612. doi:10.1111/j.1440-1746.2007.04970.x

[25] P. P. Leong, R. Mohammad, N. Ibrahim, H. Ithnin, M. Abdullah, W. C. Davis, et al., "Phenotyping of Lymphocytes Expressing Regulatory and Effector Markers in Infiltrating Ductal Carcinoma of the Breast,” Immunology Letters, Vol. 102, No. 2, 2006, pp. 229-236. doi:10.1016/j.imlet.2005.09.006 